\section{Síndrome de Cushing provocado por carcinoma suprarrenal gigante. Caso clínico}

\author{
ANTONIO ZAPATA PIZARRO ${ }^{1,2}$, MICHEL GALLEGUILLOS VALDIVIA ${ }^{1}$, \\ VÍCTOR GARCÍA JARA ${ }^{1,2}$, BALDO ESPINOZA COHEN ${ }^{1,3}$, \\ FRANKLIN ABLAN CANDIA ${ }^{1,2}$, DAVID GÜENCHOR GARCÍA ${ }^{1,4}$, \\ JOSÉ VALENZUELA CRUZ ${ }^{1,2}$.
}

\section{Cushing syndrome caused by a giant adrenal carcinoma. Report of one case}

We report a previously healthy 34-year-old woman, presenting with a seven-month history of arterial hypertension, amenorrhea, weight gain, facial edema, acne, hirsutism and low back pain. A CT scan showed a right adrenal mass of $18 \times 13 \times 12.5 \mathrm{~cm}$, and multiple vertebral and rib fractures. The hormonal study confirmed Cushing's Syndrome. Ketoconazole, spironolactone, cotrimoxazole, calcium / vitamin D were started. An adrenalectomy with a right nephrectomy were performed. The excised tumor measured $16 \mathrm{~cm}$ and weighed $1.55 \mathrm{~kg}$. There was tumor embolism and a $4 \mathrm{~mm}$ soft tissue involvement ( $p T 3 N x M x$ ). The right kidney was free of tumor. The patient was treated with chemotherapy (etoposide plus cisplatin). Study of vertebral fractures with magnetic resonance (MRI) showed crush fractures, without images of metastatic bone lesions. One year after surgery, a CT scan showed no signs of tumor recurrence. The patient was lost from follow-up thereafter.

(Rev Med Chile 2020; 148: 1679-1683)

Key words: Abdominal Neoplasms; Adrenal Gland Neoplasms; Adrenocortical Hyperfunction; Cushing Syndrome.

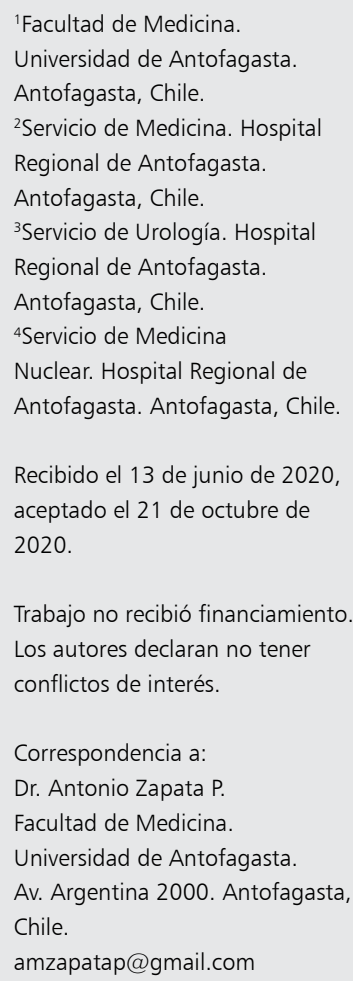

E l cáncer suprarrenal es una causa muy poco frecuente de lesión suprarrenal y de Síndrome de Cushing, originado a partir de células de la corteza suprarrenal. A menudo presenta invasión a tejidos vecinos o metástasis a distancia al diagnóstico, con sobrevida cercana a 50\% de los pacientes a 5 años. A continuación, se presenta el caso de una paciente que debutó con un hipercortisolismo severo y una gran masa abdominal, que en estudio postoperatorio correspondió a un carcinoma suprarrenal.

\section{Caso clínico}

Mujer de 34 años, sin antecedentes mórbidos. Con historia de 7 meses de evolución de com- promiso del estado general, aumento de peso, aumento de volumen facial, hipertensión arterial y amenorrea. En los últimos dos meses previos a la evaluación médica se suma aparición de acné, hirsutismo y dolor lumbar intenso. Evaluada en forma ambulatoria, se solicitó TAC (tomografía axial computada) de abdomen y pelvis que mostró gran masa sólida en polo superior renal derecho de $18 \mathrm{~cm} \mathrm{x} 13 \mathrm{~cm} \times 12,5 \mathrm{~cm}$, que borra hilio renal derecho y suprarrenal ipsilateral, esplenomegalia, colelitiasis, y fracturas costales antiguas y fracturas por aplastamiento en vertebras T5, T7 y T8 (Figura 1). Dado los hallazgos la paciente es hospitalizada y se realiza estudio de funcionalidad de masa suprarrenal, con resultados que se muestran en tabla adjunta (Tabla 1 ). 


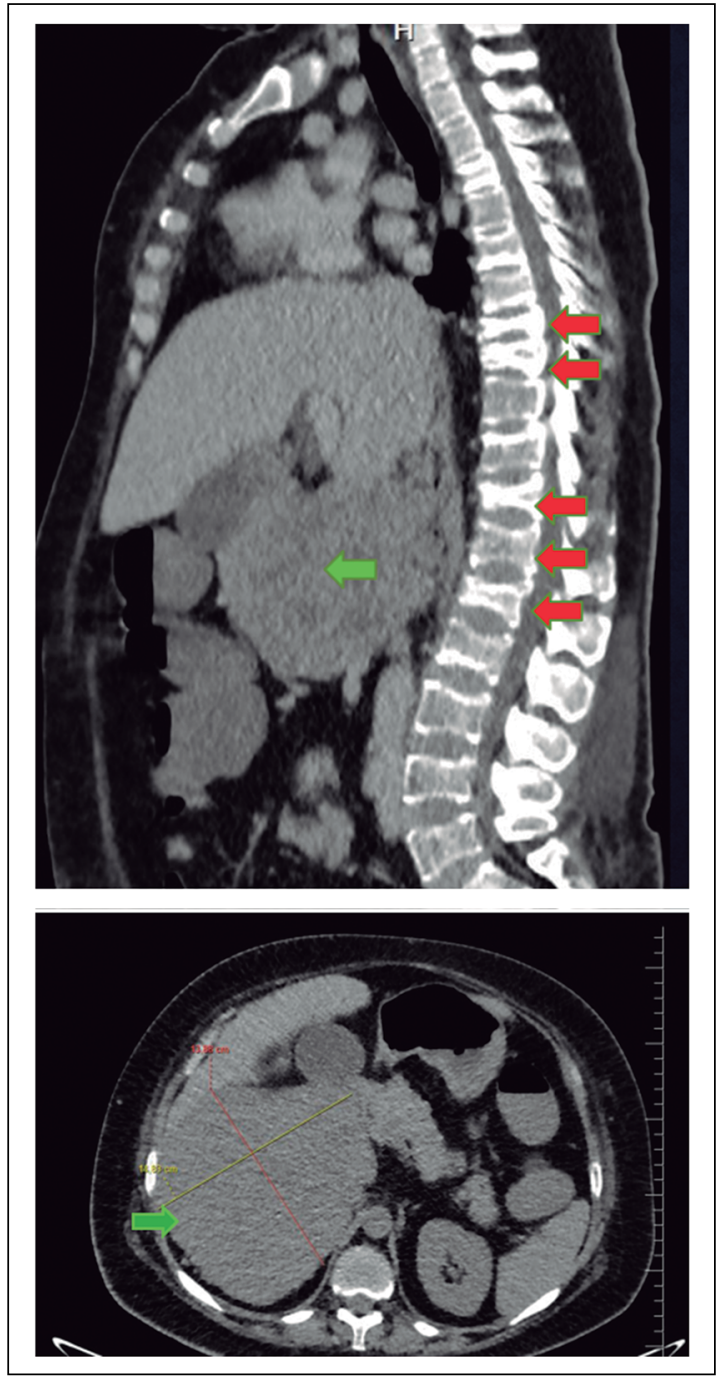

Figura 1. Reconstrucción de TAC de Abdomen. Flecha verde masa adrenal, flecha roja fracturas vertebrales.

Como complemento de estudio de etapificación, y por hallazgos de ACTH inapropiadamente normal, se solicita TAC cerebro y TAC de silla turca que fueron normales, sin anormalidad hipofisaria, pero no se logró obtener repetición de ACTH. Se realiza densitometría ósea que mostró en segmento de columna lumbar L1-L4 0,873 g/ $\mathrm{cm}^{2}(\mathrm{Z}-2,6)$, y cintigrama óseo que mostró aumento de actividad osteoblástica en T8-T9 y T11 e imágenes focales en parrilla costal bilateral compatibles con fracturas costales, pero no es posible descartar compromiso óseo 2 rio.

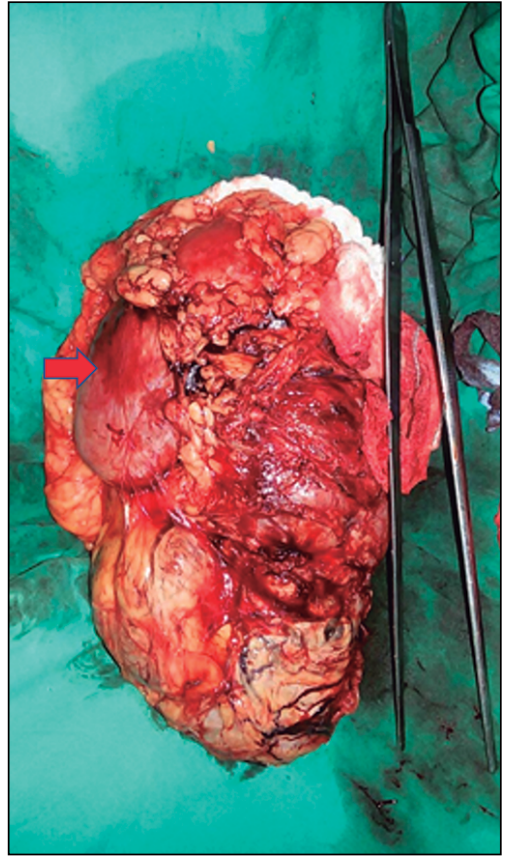

Figura 2. Flecha roja riñón, rodeado por masa tumoral adrenal. Pinza quirúrgica de referencia mide $30 \mathrm{~cm}$.

Paciente evoluciona con anasarca y dificultad respiratoria. Se inicia tratamiento con ketoconazol $200 \mathrm{mg} \mathrm{c} / 8 \mathrm{~h}$, espironolactona $25 \mathrm{mg} \mathrm{c} / 12 \mathrm{~h}$, cotrimoxazol 400/80 1 comprimido trisemanal, calcio/vitamina D 1 comprimido c/12 h. Se realiza adrenalectomía derecha con nefrectomía y colecistectomía. Se extrae, sin incidentes, masa tumoral de $16 \mathrm{~cm}$, con riñón ipsilateral, sin encontrar compromiso macroscópico de otros órganos; el tumor estaba adherido a la vena cava sin comprometerla (Figura 2). En el perioperatorio se inicia tratamiento con hidrocortisona $50 \mathrm{mg}$ c/8 h ev y luego $20 \mathrm{mg}$ AM $10 \mathrm{mg}$ PM vía oral, y se suspende ketoconazol y espironolactona. Se realiza control con exámenes de laboratorio e imagenológicos: cortisol 0,7 ug/dl (sin suplementación corticoesteroidal), TAC de tórax, abdomen y pelvis que mostró solo cambios postquirúrgicos y leve líquido en lecho quirúrgico. La biopsia definitiva mostró un carcinoma suprarrenal de $16 \mathrm{~cm}$ y $1,55 \mathrm{~kg}$, émbolos tumorales, bordes con compromiso focal de $4 \mathrm{~mm}$ en partes blandas, alto grado nuclear (pT3NxMx), riñón sin tumor. Se inicia quimioterapia con etopósido-cisplatino, dado que tratamiento de elección (mitotano) no se 
Síndrome de Cushing por carcinoma suprarrenal gigante - A. Zapata Pizarro et al

Tabla 1.

\begin{tabular}{|lcc|}
\hline Exámenes de Laboratorio & $\begin{array}{c}\text { Valores } \\
\text { preoperatorios }\end{array}$ & $\begin{array}{c}\text { Valores } \\
\text { postoperatorios* }\end{array}$ \\
\hline Actividad de renina plasmática (VN 1,2-4 ng/ml/h) & $1,96 \mathrm{ng} / \mathrm{ml} / \mathrm{h}$ \\
\hline Aldosterona (VN 2,5-16 ng/dl) & $1,96 \mathrm{ng} / \mathrm{dl}$ \\
\hline Metanefrinas en orina de $24 \mathrm{~h}(\mathrm{VN} 31-140 \mathrm{ug} / \mathrm{gr}$ creatinina) & $21 \mathrm{ug} / \mathrm{gr}$ creatinina \\
\hline Normetanefrinas en orina de $24 \mathrm{~h}(\mathrm{VN} 47-310 \mathrm{ug} / \mathrm{gr}$ creatinina) & $171 \mathrm{ug} / \mathrm{gr}$ creatinina \\
\hline Test de Nugent (VN cortisol < 1,8 ug/dl) & $40 \mathrm{ug} / \mathrm{dl}\left(1^{\circ}\right.$ examen) \\
\hline Cortisol libre urinario (VN 10-100 ug/24 h) & $42 \mathrm{ug} / \mathrm{dl}\left(2^{\circ}\right.$ examen) \\
\hline DHEA-S (VN 45-270 ug/dl) & $2.317 \mathrm{ug} / 24 \mathrm{~h}$ & $2,6 \mathrm{ug} / 24 \mathrm{~h}$ \\
Cortisol (VN 6-19 ug/dl) & $>1.000 \mathrm{ug} / \mathrm{dl}$ & $4,28 \mathrm{ug} / \mathrm{dl}$ \\
\hline ACTH (VN 6-76 pg/ml) & $39,6 \mathrm{ug} / \mathrm{dl}$ & $7,3 \mathrm{ug} / \mathrm{dl}$ \\
\hline
\end{tabular}

*Valores a los 2 meses postoperatorio con hidrocortisona suspendida el día previo a la toma de muestra.

encuentra disponible en Chile en ese momento y tampoco se pueden medir sus niveles plasmáticos. Se realiza control de laboratorio 2 meses postoperatorio, que se muestra en la Tabla 1.

En el postoperatorio, se reevalúa fracturas vertebrales con RM de columna que informa fracturas por aplastamiento en T7, T11, T12 y L3 (Figura 2), sin imágenes sospechosas de compromiso óseo secundario con efecto de masa o expansivo. El estudio del eje fosfo-cálcico demuestra normalidad (PTH 42 pg/ml, Calcemia 8,6 mg/dl, Fosfemia 3,2 $\mathrm{mg} / \mathrm{dl}$, Creatinina $0,6 \mathrm{mg} / \mathrm{dl}$, albumina $3 \mathrm{~g} / \mathrm{L})$. Se decide manejo médico de las fracturas vertebrales con corsé de Jewett. Se administra Zoledronato $5 \mathrm{mg}$ ev. Al año, con el TAC de tórax, abdomen y pelvis no muestra signos de recidiva y la densitometría ósea muestra segmento L1-L4 con 1,221g/ $\mathrm{cm}^{2}$ (score Z 0,3 vs Z-2,6 el año previo). Paciente completa ciclos de quimioterapia y deja el país, y se pierden controles médicos. Se solicitó consentimiento informado a la paciente para divulgación de sus antecedentes clínicos.

\section{Discusión}

Las glándulas suprarrenales son sitios frecuentes de aparición de lesiones nodulares. Las lesiones más frecuentes con los adenomas y las metástasis ${ }^{1}$. El cáncer suprarrenal es una causa poco común de lesión suprarrenal y etiología muy infrecuente de síndrome de Cushing. El objeto de presentar este caso clínico es ilustrar un paciente con síndrome de Cushing catastrófico inducido por un macrocarcinoma suprarrenal y discutir las opciones terapéuticas y el pronóstico de esta patología.

El carcinoma suprarrenal (CS) es una enfermedad rara, con una incidencia de 0,7 a 2 casos/ millón habitantes, más frecuente en mujeres entre 40-50 años, esporádico, pero puede asociarse a formas familiares de la enfermedad. Habitualmente el CS tiene un tamaño promedio mayor que los tumores benignos (10-12 $\mathrm{cm}$ en promedio), de hecho, el $25 \%$ de las masas adrenales $>6 \mathrm{~cm}$ son $\mathrm{CS}^{2}$. Los tumores hormonalmente funcionantes se presentan con virilización, feminización o Síndrome de Cushing, los CS no funcionantes son diagnosticados por sus manifestaciones de masa o sistémicos inespecíficos (dolor lumbar, baja de peso, etc). Habitualmente tienen mal pronóstico, con sobrevida $<30 \%$ a los 5 años ${ }^{2}$. Estudios moleculares han enumerado varios genes potencialmente involucrados en la génesis del CS (IGF2, $\beta$-catenina, TP53 ZNRF3, TERT, PRKAR1A, y otros), y algunos se asocian a síndromes genéticos familiares (Síndrome de Beckwith-Wiedemannm, Tumor de Wilms, poliposis adenomatosa familiar, síndrome de Li-Fraumenni ${ }^{3}$.

En cuanto a la presentación clínica del CS, un estudio de Abiven y col. ${ }^{2}$, de 202 pacientes con CS, mostró que 54\% de ellos fueron sintomáticos, siendo de estos la mayoría productores hormona- 
les. Guelho y col. ${ }^{4}$ en una revisión de 22 casos de CS en un período de 22 años, encontró que los principales síntomas de presentación fueron dolor lumbar, hirsutismo y aumento de peso, $50 \%$ con un estadio II en la clasificación ENSAT (European Netwotk for the Study of Adrenal Tumor $)^{5}$ al diagnóstico y en su mayoría fueron diagnosticados por TAC, muy similar al caso de nuestra paciente. En cuanto a la producción hormonal de estos tumores, la mayoría son funcionantes (52-74\%), siendo la producción mixta de cortisol y andrógenos, seguida por los productores solo de cortisol, las presentaciones más frecuentes ${ }^{2,5,6}$. Dentro de los factores de mal pronósticos del CS, los más relevantes fueron la edad, el estadio de la enfermedad y la presencia de producción de cortisol ${ }^{2,5,6}$.

Respecto al tratamiento del CS, este depende del estadío al momento del diagnóstico y del estado basal del paciente. La suprarrenalectomía es la primera elección en pacientes que no presentan metástasis (estadío I-III) y la única opción de cura. Si la resección radical es posible, la sobrevida a 5 años es de 55\%. Dada la supresión crónica que el hipercortisolismo tumoral ejerce sobre en eje hipotálamo hipófisis suprarrenal, después de la suprarrenalectomía la insuficiencia suprarrenal contralateral es de regla. Por ello es perentorio suplementar con glucocorticoides hasta que se recupere la función del eje, lo que puede demorar un año si no hay recidiva tumoral. El uso de debulking es controversial, pero podría indicarse en caso de hipercortisolismo severo para reducir la masa de células secretoras ${ }^{6}$. En referencia al tipo de cirugía, la suprarrenalectomía laparoscópica tiene menos sangrado y días de hospitalización, pero la cirugía abierta ofrece mejor exposición y resección tumoral y mayor sobrevida libre de enfermedad $^{7,8}$. Es importante considerar que la mayoría de los CS recurren, aunque hayan sido completamente extirpados; es por ello que recomienda el uso de tratamientos adyuvantes, como el mitotano (DDD), agente oral adrenolítico, que está indicado el carcinomas adrenales con alto riesgo de recurrencia (estadio III o KI $67>10 \%$ ), logra reducción de $38 \%$ de recurrencia post suprarrenalectomía en pacientes sin metástasis a distancia ${ }^{9}$; sin embargo, es un fármaco difícil de obtener y la determinación de los niveles plasmáticos son cruciales para evitar toxicidad ${ }^{5,6,9,10}$. En Chile este tratamiento no está disponible, y no existe posibilidad de medir niveles plasmáticos, por lo que no fue posible usarlo en esta paciente.

En el caso de nuestra paciente, si bien no se logró un seguimiento posterior al año de la cirugía, al parecer tuvo buena respuesta en el seguimiento, con TAC de control al año sin recidiva tumoral y desaparición de la sobreproducción hormonal, con reducción de los niveles de cortisol libre urinario en $24 \mathrm{~h}$, de DHEA-S y de cortisol basal. Es importante mencionar que el compromiso óseo que el cintigrama informaba como posibles localizaciones 2rias, hayan estado en contexto de fracturas 2rias al hipercortisolismo severo de esta paciente, como lo demostró la RM de columna. El hipercortisolismo es un factor importante de daño óseo y osteoporosis, cuya fisiopatología es multifactorial (desbalance entre de actividad osteoblástica/osteoclástica, déficit de hormona del crecimiento e hipogonadismo por efecto hipofisiario de los corticoides, alteración en metabolismo cálcico a nivel renal e intestinal, etc), que explica el compromiso en nuestra paciente tanto en la densitometrías como en las fracturas ${ }^{11}$. De hecho, es notable la variación de las densitometrías al momento de la presentación y al año postratamiento. Los niveles de ACTH inapropiadamente normal iniciales no se corresponden con el caso clínico y es muy probable que sean un error de laboratorio.

En suma, el CS es una neoplasia muy infrecuente, pero con alta morbimortalidad, que se presenta en la mayoría de las veces con síntomas secundarios al exceso de producción hormonal o síntomas compresivos locales. La suprarrenalectomía abierta es el tratamiento de elección y el uso de terapia adyuvante como el mitotano es usado en los casos de alto riesgo de recurrencia.

\section{Referencias}

1. O’Brien A, Oyanedel R, Huete A, Cruz F, Menias C. Masas Suprarrenales: Evaluación por Tomografía Computada y Resonancia Magnética. Rev Chil Radiol 2009; 15 (1): 31-8.

2. Abiven G, Coste J, Grussin L, Anract P, Tissier F, Legmann $\mathrm{P}$, et al. Clinical and biological features in the prognosis of adrenocortical cáncer: Poor outcome of cortisol-secreting tumors in a series of 202 consecutive patients. Journal of Clinical Endocrinology 2006; 91 (7): 2650-55.

3. Zheng S, Cherniack A, Dewal N, Moffitt R, Danilova L, Murray B, et al. Comprehensive pan genomic characte- 
rizacion of adrenocortical carcinoma. Cancer Cell 2016; 29 (5): 723-36.

4. Guelho D, Paiva I, Vieira A, Carrilho F. Adrenocortical carcinoma: Retrospective análisis of the last 22 years. Endocrinol Nutr 2016; 63 (5): 212-9.

5. Fassnath M, Dekkers O, Else T, Baudin E, Berruti A, de Krijger R, et al. European Society of Endocrinology Clinical Practice Guidelines of the Management of Adrenocortical Carcinoma in Adults, in collaboration with the European Network for the Study of Adrenal Tumors. Eur J Endocrinol 2018; 179: G1-G46.

6. Puglisi S, Perotti P, Pia A, Reimondo G, Terzolo M. Adrenocortical carcinoma with hypercortisolism. Endocrinol Metab Clin N Am 2018; 47 (2): 395-407.

7. Zheng G, Li H, Deng J, Zhang X, Wu X. Open adenalectomy versus laparoscopic adrenalectomy for adrenocortical carcinoma: a retrospective comparative study on short-term oncologic prognosis. Onco Targets Ther
2018; 11: 1625-32.

8. Conzo G, Tartaglia E, Gambardella C, Esposito D, Sciascia V, Mauriello C, et al. Minimally invasive approach for adrenal lesions: Systematic review of laparoscopic versus retroperitoneoscopic adrenalectomy and assessment of risk factors for complications. Int J Surg 2016; 1: S118-123.

9. Tang Y, Liu Z, Zou Z, Liang J, Lu Y, Zhu Y. Benefits of adjuvant mitotane after resection of adrenocortical carcinoma: A systematic review and meta-analysis. Biomed Research International. 2018. Article ID 9362108, 8 pages, 2018.

10. Berruti A, Naudin E, Gelderblom H, Haak H, Porpiglia F, Fassnacht M, et al. Adrenal cancer : ESMO Clinical Practice Guidelines . Ann Oncol 2012; 23 (suppl 7): 131-8.

11. Compston J. Glucocorticoid-induced osteoporosis: an update. Endocrine 2018; 61 (1): 7-16. 